\title{
SGRs and AXPs: Evidence for Delayed Amplification of Magnetic Field after Neutron Star Formation?
}

\author{
Denis Leahy and Rachid Ouyed \\ Department of Physics and Astronomy, University of Calgary, 2500 University Drive NW, Calgary, AB, Canada T2N 1N4 \\ Correspondence should be addressed to Denis Leahy, leahy@ucalgary.ca
}

Received 13 November 2008; Revised 1 April 2009; Accepted 12 May 2009

Recommended by Valentina Klochkova

\begin{abstract}
We present new analysis of the birth rate of AXPs and SGRS and their associated SNRs. Using Kolmogorov-Smirnov statistics together with parametric fits based on a robust estimator, we find a birth rate of $\sim 1 /(1000$ years $)$ for AXPs/SGRs and their associated SNRs. These high rates suggest that all massive stars (greater than $\sim(23-32) M_{\odot}$ ) give rise to remnants with magnetarlike fields. Observations indicate a limited fraction of high magnetic fields in these progenitors; thus our study is suggestive of magnetic field amplification. Dynamo mechanisms during the birth of the neutron stars require spin rates much faster than either observations or theory indicate. We propose that massive stars produce neutron stars with normal $\left(\sim 10^{12} \mathrm{G}\right)$ magnetic fields, which are then amplified to $10^{14}-10^{15} \mathrm{G}$ after a delay of hundreds of years. The amplification is speculated to be a consequence of color ferromagnetism and to occur with a delay after the neutron star core reaches quark deconfinement density (i.e., the quarknova scenario). The delayed amplification allows one to interpret simultaneously the high birth rate and high magnetic fields of AXPs/SGRs and their link to massive stars.
\end{abstract}

Copyright (C) 2009 D. Leahy and R. Ouyed. This is an open access article distributed under the Creative Commons Attribution License, which permits unrestricted use, distribution, and reproduction in any medium, provided the original work is properly cited.

\section{Introduction}

Early studies of association of Anomalous X-ray Pulsars (AXPs) with supernova remnants (SNRs) suggested that $5 \%$ of core-collapse SN results in AXPs [1]. This was based on 3 SNR associations out of a total of 6 AXPs. Since then evidence has mounted that AXPs and soft gamma-ray repeaters (SGRs) are the same type of objects [2] and more AXPs, SGRs and associated SNRs have been identified. Thus it is timely to revisit the issue of AXPs/SGRs birthrates.

In this study we present an updated investigation of the birth rate of AXPs/SGRs and in addition, the birth rate of associated SNRs is given. Since AXPs/SGRs ages rely on spin-down age estimates whereas SNRs ages are based on shock expansion models, this constitutes two independent estimates for birth rates. We find here that both samples yield a high birth rate for AXPs/SGRs of (1/5)-(1/10) of all core-collapse $\mathrm{SNe}$, higher than previously appreciated. (An independent study by Gill and Heyl [3], based on a population synthesis of AXPs detected in the ROSAT AllSky Survey, yields a birth rate of $\sim 0.22$ per century.) This high frequency of occurrence of AXPs/SGRs brings into focus issues related to the origin of the strong magnetic fields which we address here. This paper is presented as follows. Section 2 describes the methods and presents the birth rate results, and Section 3 discusses the implications. Our model, based on a delayed amplification of magnetic field, is presented in Section 4 before concluding in Section 5 .

\section{Birth Rate Based on Spin-Down and SNR Ages}

We start by analyzing the age distribution of AXPs/SGRs and their associated SNRs. One can derive birth rates by fitting a linear trend to the observed cumulative number versus age relation. (The cumulative age distribution is the steplike function with unit step along the $y$-axis and age along the $x$-axis. This becomes the cumulative age probability distribution upon dividing by the total sample size.) For SNRs, we take data from Marsden et al. [4] and Gaensler et al. [5] and for spin-down ages we used $P$ and $\dot{P}$ from the 
TABLE 1: SGRs/AXPs data mainly from Gaensler et al. [5], Marsden et al. [4], the atnf pulsar catalog [12], and Camilo [13]; with updates as indicated by footnotes. Spin-down ages, $\boldsymbol{\tau}_{\mathrm{SD}}$, and SNR ages, $\boldsymbol{\tau}_{\mathrm{SNR}}$, in years.

\begin{tabular}{|c|c|c|c|c|c|c|c|c|}
\hline Source & $\tau_{\mathrm{SD}}$ & $\tau_{\mathrm{SNR}, \text { lower }}$ & $\tau_{\mathrm{SNR} \text {,upper }}$ & $\tau_{\mathrm{SNR}, \text { mean }}$ & Assoc. ${ }^{\dagger}$ & $d_{\mathrm{kpc}}$ & $v_{\mathrm{SNR}}(\mathrm{km} / \mathrm{s})$ & $v_{\mathrm{SD}}(\mathrm{km} / \mathrm{s})$ \\
\hline SGR1806-20 & 281 & 3500 & 30000 & 10300 & $S^{1}(<1 \%)$ & $15^{2}$ & 100 & 3800 \\
\hline SGR1900+14 & 1050 & 9600 & 30000 & 17000 & $A^{3}(4 \%)$ & 7 & 2000 & 32000 \\
\hline SGR0525-66 & 1960 & 5000 & 16000 & 8940 & $\mathrm{~S}(0.2-0.7 \%)$ & 50 & 800 & 3600 \\
\hline SGR1627-41 & N/A & 2600 & 30000 & 8830 & $A^{4}(5 \%)$ & 11 & 460 & N/A \\
\hline AXP1E1841-045 & 4510 & 500 & 2500 & 1120 & S $(0.01 \%)$ & 7 & $<890$ & $<220$ \\
\hline AXP1845-0258 & N/A & 600 & 30000 & 4240 & $S(0.2 \%)$ & $<20$ & $<870$ & N/A \\
\hline AXP1E2259+586 & 228000 & 3000 & 17000 & 7140 & S $(0.05 \%)$ & $3^{7}$ & $<360$ & $<11$ \\
\hline AXP1E1048-5937 & $3780^{5}$ & N/A & N/A & N/A & N/A & N/A & N/A & N/A \\
\hline AXPRXS1708-4009 & 8960 & N/A & N/A & N/A & N/A & N/A & N/A & N/A \\
\hline TAXPXTEJ1810-197 & $11300^{6}$ & N/A & N/A & N/A & N/A & N/A & N/A & N/A \\
\hline TAXPJ0100-7211 & 6760 & N/A & N/A & N/A & N/A & N/A & N/A & N/A \\
\hline AXP1547-5408 & 1400 & N/A & N/A & N/A & N/A & N/A & N/A & N/A \\
\hline AXP4U0142+615 & 70200 & N/A & N/A & N/A & N/A & N/A & N/A & N/A \\
\hline
\end{tabular}

${ }^{1}$ Frail et al. [14]; ${ }^{2}$ McClure-Griffiths and Gaensler [15]; ${ }^{3}$ Vasisht et al. [16]; Lorimer and Xilouris [17]; ${ }^{4}$ Smith et al. [18]; ${ }^{5}$ based on $\dot{\nu}$ prior to 2002 outburst [19]; ${ }^{6}$ Camillo et al. [20]; ${ }^{7}$ Kothes et al. [21].

${ }^{\dagger} \mathrm{S}$ for secure and $\mathrm{A}$ for ambiguous association (random association probability given in brackets); ${ }^{\ddagger}$ unconfirmed AXP.

Australia Telescope National Facility (ATNF) website, with individual cases updated from literature (see Table 1). The sample consists of 4 SGRs and 9 AXPs and 7 associations with SNRs. In Table 1, we list the associations as either secure (S) or ambiguous (A). Secure associations are those where the chance overlap probability $<1 \%$, and the ambiguous associations have chance overlap probabilities $<5 \%$ [5]. The poor quality associations ( $>5 \%)$ were omitted. Given the $5 \%$ limit, the four ambiguous associations are unlikely to contain more than one false association; so we include all four in our statistical analysis. For the mean SNR ages we adopt the geometric mean of the lower and upper limits.

From Table 1, AXP1E2259+586 and AXP4U0142+615 which have spin-down ages are much longer than all other AXPs/SGRs (of order 10 to $>200$ times longer), yet they have similar X-ray luminosities. Their ages are inconsistent with birth rates based on the other AXPs/SGRs, so we believe that they stand out as being different objects in some fundamental way. Ertan et al. [6] give theoretical and observational arguments for a disk around AXP1E2259+586; they argue that the disk is needed to explain infrared and X-ray observations. As for AXP4U0142+615, Alpar [7] and Chatterjee et al. [8] argue for a nonpassive disk. Our previous work [9] presents an alternate model also involving debris material surrounding the quark star. In our model accretion from degenerate iron rich debris provides the Xray luminosity. Thus our proposal for these two objects is that they differ because they have active accretion (i.e., from degenerate iron rich debris as explained by Ouyed et al. $[9,10])$ that changes their spin-down history. Objects AXP1E2259+586 and AXP4U0142+615 are further discussed in Section 4.1 here. Thus we do not use these two spin-down ages.

We apply the Kolmogorov-Smirnov (KS) statistical test [11] to test whether simple models such as a constant age model and a constant birth-rate model are consistent with observations. To apply the KS test both the data and the models are converted to probability distributions by normalizing. Then the maximum difference is used in the KS test. In Figure 1 we show the cumulative age probability distributions for SNR age (a) and spin-down age (b), together with the expected distributions for constant age or constant birth rate. For the constant age hypothesis we choose the age that minimizes the discrepancy with the observed distribution, that is, SNR age of 8800 years and spin-down age of 3800 years. We find that the constant age hypothesis is weakly rejected (null hypothesis probability $11 \%$ ) whereas the constant birth rate hypothesis is not rejected (null hypothesis probability 69\%). For the spin-down ages, we find that the constant age hypothesis is rejected (null hypothesis probability 4\%) whereas the constant birth rate hypothesis is not rejected (null hypothesis probability $54 \%$ ). There is a clustering of points in Figure 1(a); three SNR ages are clustered in a narrow range from 8800 to 10300 years. The KS clarifies this issue: the ages are significantly more consistent with constant birth rate than with constant age hypothesis.

In order to derive a birth rate from the data we go beyond the nonparametric KS test and carry out model fitting: one set of fits assumes normal statistics and another set uses a robust estimator (e.g., Press et al. [11, Section 14]). The robust estimator uses the sum of absolute values of differences rather than the sum of squares and thus gives less weight to outliers. For the SNR ages, the error was taken as proportional to the difference between the maximum and minimum SNR ages. For the SD ages, the error was taken as proportional to the SD age. The constant of proportionality has no effect on the best-fit birth rate. The results assuming normal statistics give an SGR/AXP birth rate from spin-down of $1 /(480$ years $)$ with a $1 \sigma$ range from $1 /$ (390 years) to $1 /(580$ years $)$, assuming a $50 \%$ uncertainty in spin-down age. For the robust estimator the best fit is $1 /(490$ years) consistent with the above. For the 


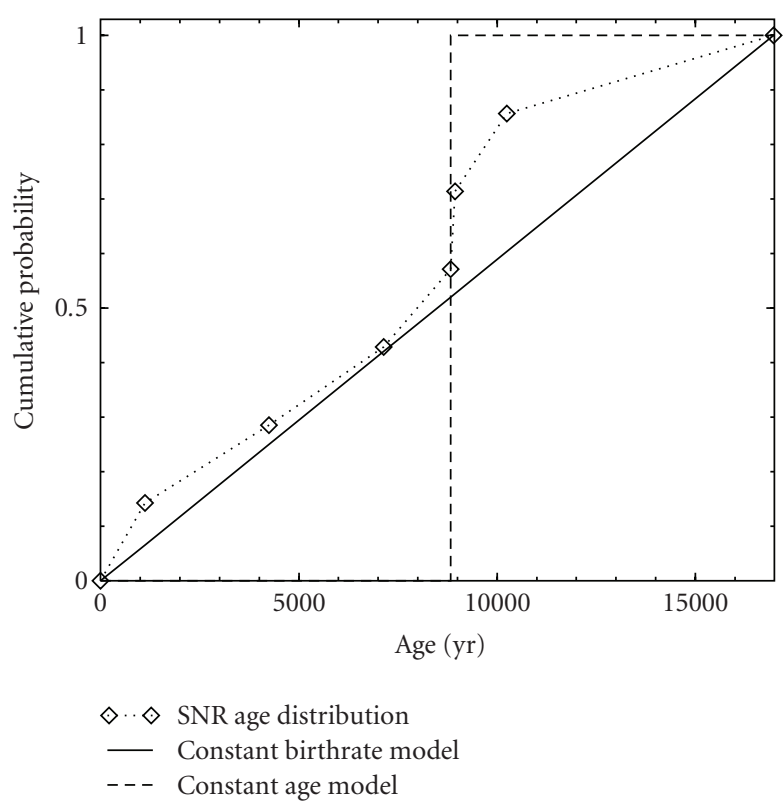

(a)

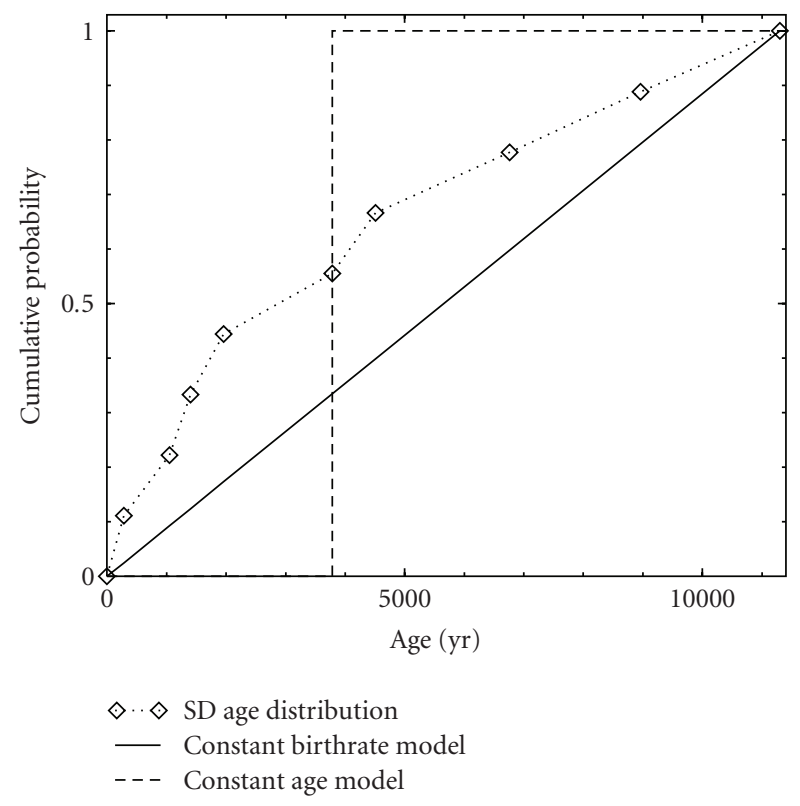

(b)

FIGURE 1: (a) shows the cumulative probability distribution for ages of SNRs associated with AXPs/SGRs (diamonds). Also shown is the expected probability distributions for the cases of constant birth rate (solid line) and of constant age (dashed line). (b) shows the cumulative probability distribution for spin-down ages of AXPs/SGRs (diamonds) and expected probability distributions for constant birth rate (solid line) and of constant age (dashed line) models. The ages for the constant age models were chosen to give minimum KS statistic (and lowest rejection probability) for both SNR and spin-down ages.

associated SNRs, the resulting birth rate from the normal estimator is $1 /(1700$ years $)$ with a $1 \sigma$ range from $1 /(1500$ years) to $1 /(1900$ years $)$ while the robust estimator gives $1 /(1800$ years $)$. In all cases the $\chi^{2}$ values were acceptable indicating that a constant birth rate fit is an acceptable model.

Figure 2(a) shows the cumulative number of associated SNRs ( $N_{\mathrm{SNR}}$, diamonds and dot-dashed line) versus SNR age. Since there are a wide range of age and only a few objects $(<10)$, we plot number on a linear scale and age on a logarithmic scale, as a result a linear relation looks curved. To show the uncertainties in the ages we also plot the minimum and maximum ages for each SNR. The solid line is the expected number versus age relation (i.e., $N=B \times t$ ), with $B=1 /(1700$ years $)$ the birth rate constant. In Figure $2(b)$ we show the cumulative number of SGRs/AXPs ( $N_{\mathrm{SD}}$, circles and dashed line) versus spin-down age. The solid line is the expected number versus age relation for a constant birth rate of 1/(500 years). In Figure 2(b) we replot the cumulative number versus age relation for associated SNRs scaled up by a factor of 3 ( $3 N_{\mathrm{SNR}}$, diamonds and dotted line); the dotdashed line replots the SNR birth rate of 1/(1700 years) from Figure 2(a).

2.1. Birth Rate Comparison. The birth rate derived from associated SNRs is $\sim 1 / 3$ of the birth rate derived from spindown ages. There are two effects that could account for such a discrepancy.
One effect is incompleteness of either sample, which would increase the birth rate of that sample; in this case incompleteness of the SNR sample could increase the birth rate to match the birth rate from spin-down. As can be seen from Figure 2(b), if we increase the number of SNRs by a factor of about 3, we obtain good agreement with the number versus age relation for AXPs/SGRs. It is worth pointing out that, of the $13 \mathrm{AXPs} / \mathrm{SGRs}, 7$ show associated SNRs. Since all AXPs/SGRs have been searched for associated SNRs, the SNRs are too faint to be seen. This is either due to (i) the SNR is old; (ii) the SNR is not detected due to confusion; (iii) the SNR is young but the environment has low density. Either of the latter two situations suggests incompleteness, with a factor of about $\sim 13 / 7$, raising the birth rate estimate from associated SNRs to $\sim 1 /(900$ years); this is not enough to account for the difference in birth rates. For SGRs/AXPs birth rate if there is incompleteness in the sample, then the birth rate increases above $1 /$ (500 years). However, these objects are fairly bright in X-rays so only transient SGRs/AXPs would contribute to incompleteness. The high birth rate we derived indicates that there cannot be very many $(<10)$ transients. However there could be a large number of transients if they do not produce observable SNRs, which we do not consider here. With the above assumption, the incompleteness should not be an important factor otherwise we overproduce AXPs/SGRs compared to the total SN rate in the Galaxy.

The second effect is that SNRs or AXPs/SGRs spin-down ages could be systematically off by a factor of $\sim 2$. In effect 


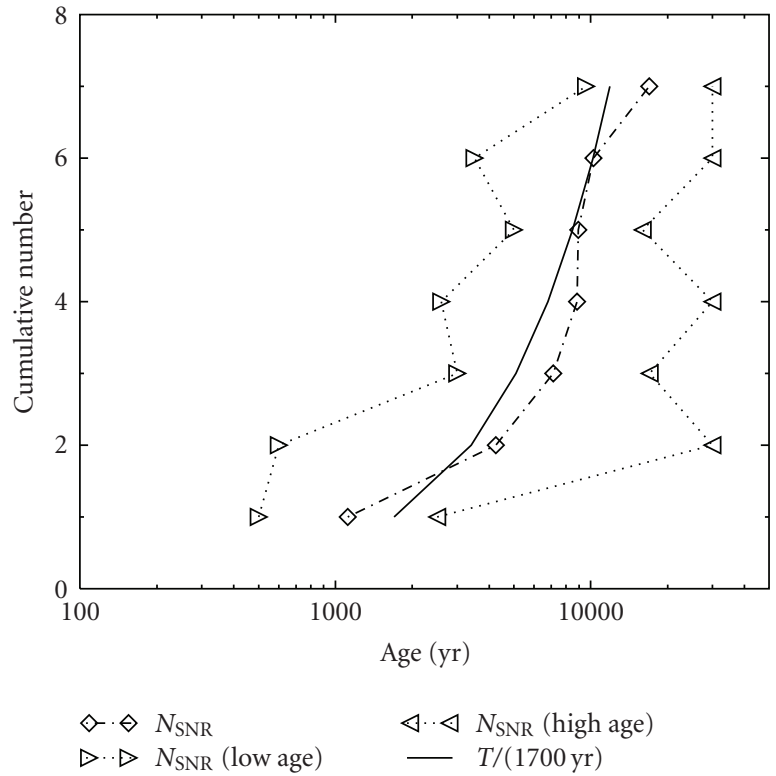

(a)

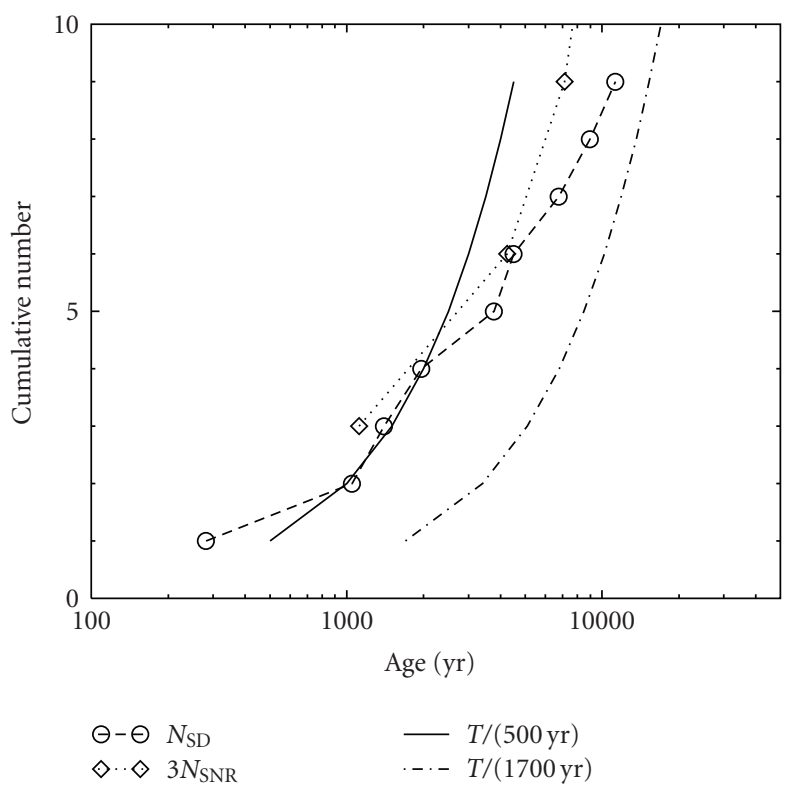

(b)

FIGURE 2: (a) shows the cumulative age distribution for SNRs associated with AXPs and SGRs (diamonds and dot-dashed line). The solid line is the expected distribution for constant birth rate of $1 /(1700$ years). The triangles indicate the upper and lower SNR age limits corresponding roughly to $3 \sigma$ limits. (b) shows the cumulative age distribution for AXPs/SGRs (circles and dashed line). The solid line is the expected distribution for constant birth rate of 1/(500 years). The diamonds and dotted line are the cumulative distribution for associated SNRs (from (a)) scaled up by a factor of 3 . To better illustrate this scaling, the $1 /(1700$ years) line is replotted (dot-dashed line). Systematic errors in spin-down age would shift the $x$-axis and are in Section 2.1.

instead of shifting points vertically in Figure 2, the points are shifted horizontally. There is no reason why the SNR ages should be systematically too large by up to a factor of $\sim 2$. However there are reasons to believe that spin-down ages may systematically be off. The general spin-down formula for braking index $n, \dot{\Omega}=-K \Omega^{n}$ (where $K$ is a constant; e.g., [22]), implies a spin-down age $\tau=P /((n-1) \dot{P})$. Table 1 assumes the vacuum dipole case with $n=3$. However the few pulsars with measured braking indices have values $n>2$ with the exception of the Vela pulsar with $n=1.4$ [23]. For $n=2$ the spin-down age is twice that listed in Table 1: this can bring the spin-down derived birth rate down to $\sim 1 /(1000$ years) in agreement with the SNR derived value corrected for incompleteness.

However, for 3 of the objects listed (SGR1806-20, SGR1900+14, and SGR0525-66) a doubled spin-down age is still not enough to remove the discrepancy between spindown age and the lower limit to the SNR age. (Park et al. [24] give a recent estimate of 6600 years for the age of N49, associated with SGR0525-66-this age is near the lower limit of earlier estimates (see Table 1). The spin-down age of 1960 is still less than the SNR age by a substantial amount.) If the initial period of the neutron star is below $\sim 1 \mathrm{~ms}$, the moment of inertia decreases as it spins down (e.g., [25]). The spin-down formula given above assumes constant moment of inertia and thus a constant $K$. Taking into account changes in the oblateness (moment of inertia) as the star spin-down from millisecond period leads to no more than $20 \%-30 \%$ increase in age estimate. This is not large enough to explain the discrepancy. On the other hand, spin-down ages assume constant magnetic field. Including magnetic field decay will decrease these ages and worsen the discrepancy. (Bhattacharya and Soni [26] propose high internal magnetic fields caused by pion condensate core inside a neutron star; the magnetic field emerges at the surface later in the evolution of the neutron star due to the effect of early screening currents in the crust.)

To summarize this section, the AXPs/SGRs birth rate from spin-down is about $1 /(500$ years) $(n=3)$ to $1 /(1000$ years) $(n=2)$. The latter is consistent with the SNR-derived rate corrected for incompleteness. We still need to explain a large discrepancy in age for the three cases mentioned above. We suggest a time delay from SNR explosion to the onset of spin-down to explain these cases (see Section 4).

\section{Implications}

We consider the implications of the high birth rate. A birth rate of $1 /(500$ years $)$ to $1 /(1000$ years $)$ implies $1 / 5$ to $1 / 10$ of all core-collapse SNe lead to SGRs/AXPs. To interpret this we use a Scalo mass function, minimum and maximum SN progenitor masses of $9 M_{\odot}$ and $60 M_{\odot}$. For the $1 / 5$ case, the $\mathrm{SGR} / \mathrm{AXP}$ progenitor mass range is from $23 M_{\odot}$ to $60 M_{\odot}$; for the $1 / 10$ case, the SGR/AXP progenitor mass range is from $32 M_{\odot}$ to $60 M_{\odot}$. The ranges can be shifted as long as they give the same fraction of SNe that lead to SGRs/AXPs (e.g., from $20 M_{\odot}$ to $40 M_{\odot}$ for the $1 / 5$ case). 
An alternate possibility is that $1 / 5$ to $1 / 10$ of $\mathrm{SNe}$ for all progenitor masses produce AXPs/SGRs. However observations of associated SNRs indicate that SGRs/AXPs are associated with massive star progenitors [1], so we favor $100 \%$ production at the high-mass end with $M>M_{\text {low }}=$ (23-32) $M_{\odot}$.

This raises the following questions.

(1) How do all progenitors with $M \geq M_{\text {low }}$ generate greater than $10^{14} \mathrm{G}$ fields in their compact remnants?

(2) Why is there a sudden jump in the magnetic field strength between compact remnants from progenitors with mass greater than $M_{\text {low }}$ (i.e., $B \sim 10^{14} \mathrm{G}$ ) and those with mass less than $M_{\text {low }}\left(B \sim 10^{12} \mathrm{G}\right)$ ?

In regards to point (1), observations of $\mathrm{OB}$ stars [27] found 3 out of 8 with $\sim \mathrm{kG}$ fields and one out of two massive stars with $\sim \mathrm{kG}$ fields. Despite the paucity of data, this indicates that about half of massive stars are strongly magnetic. The fossil field hypothesis [28] predicts that a high magnetic field progenitor produces a magnetar and a low magnetic field progenitor produces a low (or normal) field neutron star. Our limited statistical study suggests that all massive progenitors, whether magnetic or nonmagnetic, should produce magnetars. This implies that a magnetic field amplification mechanism is required to explain the high fields in all compact remnants from massive progenitors. Point (2) further suggests some new physical mechanism for magnetic field amplification that sets in, independent of progenitor magnetic field, but dependent on progenitor mass.

One natural mechanism would be dynamo generation during neutron star formation [29]. However as shown by Vink and Kuiper [30] the SNRs associated with SGRs and AXPs have normal explosion energy $\left(\sim 10^{51} \mathrm{erg}\right)$ conservatively limiting the birth periods to $>5 \mathrm{~ms}$. This provides a major challenge for the dynamo mechanism for the generation of SGR/AXP magnetic field strengths. Heger et al. [31] also consider the spin periods of neutron stars at birth from massive stars using a stellar evolution code. They calculate the evolution of $12-35 M_{\odot}$ progenitors including magnetic field and angular momentum transport. Their Table 4 gives results of $\sim 15 \mathrm{~ms}$ (for $12 M_{\odot}$ ) to $3 \mathrm{~ms}$ (for $35 M_{\odot}$ ), many times slower than previously obtained in calculations ignoring magnetic torques. This in effect also argues against the dynamo mechanism, which requires sub$\mathrm{ms}$ periods, to generate magnetar-like fields for stars of $35 M_{\odot}$ or less. This leaves us with the dilemma: how to account for the strong magnetic fields inferred for AXPs/SGRs (i.e., all descendants of stars are more massive than $\left.M_{\text {low }}\right)$ ?

In summary, our reasoning is as follows: (i) high birth rates of magnetars imply that all massive stars with $M>$ $M_{\text {low }}$ produce magnetars; (ii) observations of massive stars imply need of magnetic field amplification mechanism; (iii) observations of associated SNRs imply that the dynamo mechanism is unlikely to cause the amplification; (iv) we propose (see next) delayed magnetic field amplification associated with delayed quark star formation after neutron star birth.

\section{Proposed Explanation}

We offer an alternate explanation which allows normal magnetic fields for neutron stars born from progenitors with mass $>M_{\text {low }}$, in addition to lower mass progenitors. In this picture, the magnetic field amplification occurs long after the neutron star formation, but only for neutron stars born from massive progenitors. The amplification occurs during the conversion from baryonic matter to quark matter which happens after the neutron star core reaches quark deconfinement density.

Amplification of the magnetic field up to $10^{15} \mathrm{G}$, or greater, can be achieved as a result of color-ferromagnetism [32] during the phase transition. The magnetization here is unlike the case of a normal ferromagnet where spontaneous magnetization occurs as the temperature falls below the Curie temperature (there the order parameter is the spontaneous magnetization $M$ namely, the expectation value of spin over the sample, and an external field is required to impose domain alignment). Color-ferromagnetism instead is dictated by the Savvidy effect which is an instability of the vacuum due to infrared singularities [33]. In ColorFerromagnetic quark matter (SU(2)), the color magnetic field is generated spontaneously not by alignment of quark color spins, but by the dynamics of the gluons [34]. Due to the nature of fractional quantum hall states, the color magnetic field can exist globally in the quark matter, without domain structure. This global uniform field is the minimum energy state (see [32], and references therein).

Deconfinement can be delayed from the time of formation of the neutron star by two mechanisms. First, there is a delay between neutron star birth and the time deconfinement density is reached. The proto-neutron star cools rapidly slightly increasing its density but a much more effective mechanism to increase the central density exists for rotating neutron stars [35]. Second, a delay exists between the time that deconfinement density is reached and the time that conversion of hadrons to quarks is complete (e.g., [36]). We refer to the first as spin-down delay and to the second as conversion delay. Regarding the second delay mechanism, the conversion delay, subsequent to reaching deconfinement density, is independent of spin period. Thus such a delay is expected also for slowly rotating neutron stars.

Staff et al. [35], neglecting conversion delay, discuss the time delay from neutron star formation to deconfinement density in the core and subsequent quark star formation (which occurs in an explosive manner, called a QuarkNova or QN; Ouyed et al. [37]; Keränen and Ouyed [38]; Keränen et al. [39]). They found that neutron stars with (i) mass greater than $\sim 1.5 M_{\odot}$, (ii) initial periods less than $\sim 3 \mathrm{~ms}$ and (iii) magnetic fields less than $\sim 10^{12} \mathrm{G}$, experience deconfinement with typical delays of several hundred years (see Table 2 and discussion by Staff et al. [35]). These numbers are interesting as they imply progenitors consistent with those we discussed above in the context of birth rates of AXPs/SGRs (i.e., $M>M_{\text {low }}, B \sim 10^{12} \mathrm{G}$ and, periods of a few milliseconds). For more massive neutron stars (with progenitors mass around approximately $\sim 50-60 M_{\odot}$ ) the 
delay is days rather than centuries leading to an energized SN instead [40]. More massive progenitors lead to black holes.

The energy loss rate from the young neutron star is given by

$$
\dot{E}_{\text {dip. }} \propto \frac{B^{2}}{P^{4}},
$$

where $B$ is the magnetic field and $P$ the spin period. Since neutron stars with spin-down delays of a few hundred years require normal magnetic fields $\left(\lesssim 10^{12} \mathrm{G}\right)$ in our model, there is no over-energetic central pulsar in the SNR. This is consistent with observations of Vink and Kuiper [30]. In contrast the magnetar model, with the magnetic field generated by the dynamo mechanism, requires a $B \sim 10^{15} \mathrm{G}$ and $P<1 \mathrm{~ms}$, implying energies loss rates $\sim 10^{7}-10^{8}$ larger than in our model.

To represent the general idea of a delayed amplification of the magnetic field, we write the time since SN explosion as

$$
\tau_{\mathrm{SNR}}=\tau_{\mathrm{NS}}+\tau_{\mathrm{QS}}
$$

where $\tau_{\mathrm{NS}}$ and $\tau_{\mathrm{QS}}$ are the time the compact object spends as a neutron star and quark star, respectively. In our model the magnetic field during the neutron star era $\left(\tau_{\mathrm{NS}}\right)$ is $\sim 10^{12} \mathrm{G}$ thus spin-down is slow during this period. The delay time, $\tau_{\mathrm{NS}}$, is defined by the spin-down delay plus the conversion delay. After deconfinement and associated QN, the object's magnetic field is strongly magnified leading to a fast spindown. The result is that the spin-down age, $\tau_{\mathrm{SD}}$, is given by $\tau_{\mathrm{SD}}=\tau_{\mathrm{QS}}$, which is determined by vortex expulsion and associated magnetic field decay [41-44]. As shown by Ouyed et al. [10], the resulting spin-down age is $0.16 P / \dot{P} \leq \tau_{\mathrm{SD}} \leq$ $0.33 P / \dot{P}$. This is reduced with respect to the standard value $P /(2 \dot{P})$ by a factor of $\simeq 1.5-3$.

From Table 1, as noted above, 3 objects have spin-down ages much less than the minimum associated SNR age. Of these, SGRs 1806-20 and SGR 0525-66 have good associations while SGR $1900+14$ has an ambiguous association. As a further test we added the transverse velocities based on offsets from the SNR centers (see Table 1). As can be seen the transverse velocities using SNR ages for all cases except SGR $1900+14$ are reasonable. This suggests that the SGR $1900+14$ association is not real but the remaining 2 are reasonable. Thus we think that we have 2 good cases of $\tau_{\mathrm{SD}} \ll \tau_{\mathrm{SNR}}$. The delayed amplification of magnetic field can alleviate this problem, that is, 3000 years $<\tau_{\mathrm{NS}}<9000$ years and 200 years $<\tau_{\mathrm{SD}}<2000$ years.

The only object in Table 1 that has spin-down age reliably greater than SNR age is AXP1E1841-045: the reduced spindown age in our model becomes consistent with the SNR age. In this case $\tau_{\mathrm{NS}} \ll \tau_{\mathrm{QS}}$, thus no delay is required.

We have argued above that standard spin-down age estimates do not represent true ages. This affects the birth rate estimate for SGRs/AXPs given above in two ways. Firstly, the spin-down era is shorter by a factor of $\sim 1.5-3$ or an average of 2.25. Secondly, the time since SN explosion is lengthened by the time delay to magnetic field amplification. In reality the delay time is different for each object depending on the neutron star's initial period, magnetic field, and mass. As an approximation, we carried out fits with new age estimates using (2) with fixed $\tau_{\mathrm{NS}}$, and with $\tau_{\mathrm{QS}}=$ $\tau_{\mathrm{SD}} / 2.25$. For $\tau_{\mathrm{NS}}=200,500,1000,3000$ years, the resulting birth rates were $1 /(316$ years $), 1 /(400$ years $), 1 /(510$ years $)$, $1 /(875$ years $)$, respectively. Thus our previous estimates of $1 /(500$ years $)$ to $1 /(1000$ years $)$ is valid but the uncertainty is increased. This does not affect the main conclusion that about $1 / 5$ to $1 / 10$ of all core-collapse $\mathrm{SN}$ results in AXPs/SGRs.

4.1. AXPs 1E2259+586 and 4U0142+615. Alpar [7] suggested that all AXPs/SGRs have normal magnetic fields $\left(\sim 10^{12} \mathrm{G}\right)$. (Instead Dar and DeRújula [45] suggest a conversion to quark matter accompanied by a slow gravitational contraction to power the observed emission.) The large spin-down rates of AXPs/SGRs are then explained by accretion (with propeller mechanism to give the large positive $\dot{P}[8]$ ) from a fall-back disk following the SN explosion. However, there are several destruction mechanisms such as radiation, magnetic propeller and flares that probably limit the lifetime of such disks. Instead in our model, a tiny degenerate iron-rich shell [10] or a Keplerian torus [9] forms from the ejected neutron star crust following a Quark-nova. This degenerate matter can survive the destruction mechanisms that act on a nondegenerate disk.

For AXPs 1E2259+586 and 4U0142+615 we found that their X-ray luminosity is determined by accretion from the torus. Thus their age as quark stars is $\tau_{\mathrm{QS}} \neq \tau_{\mathrm{SD}}$. This may explain the absurdly high spin-down age for 1E2259+586 compared to its associated SNR age. We have previously argued that the same situation applies to $4 \mathrm{U} 0142+615$ (see Figure 1 of Ouyed et al. [10]). The estimate of $\tau_{\mathrm{OS}}$ is the time it takes to consume the torus or, $\tau_{\mathrm{QS}} \sim m_{\mathrm{t}} / \dot{m}_{\mathrm{t}, \mathrm{q}}$. The continuous (i.e., quiescent phase) accretion rate, $\dot{m}_{\mathrm{t}, \mathrm{q}}$ is given by equation (20) of Ouyed et al. [9] (see also Ouyed et al. [46]). We find

$$
\tau_{\mathrm{QS}} \simeq 1.6 \times 10^{4} \text { years } \frac{m_{\mathrm{t},-7} M_{\mathrm{QS}, 1.4}^{4} \mu_{\mathrm{q}, 3.3}^{6}}{\eta_{0.1}^{3} R_{\mathrm{t}, 15}^{6}},
$$

where $m_{\mathrm{t},-7}$ and $R_{\mathrm{t}, 15}$ are the mass and radius of the torus in units of $10^{-7} M_{\odot}$ and $15 \mathrm{~km}$, respectively, $M_{\mathrm{QS}, 1.4}$ is the mass of the quark star in units if $1.4 M_{\odot}, \mu_{\mathrm{q}} \sim 3.3$ is the mean molecular weight of the torus atmosphere, and $\eta_{0.1}$ is the accretion efficiency in units of 0.1 . Referring to (2), there is no longer a discrepancy between the calculated $\tau_{\mathrm{SNR}} \sim \tau_{\mathrm{QS}}$ and the observed SNR age given $\tau_{\mathrm{NS}} \ll \tau_{\mathrm{QS}}$.

\section{Conclusion}

Our study of the birth rate of AXPs and SGRS and their associated SNRs suggests that about $1 / 5$ to $1 / 10$ of all corecollapse SN leads to AXPs/SGRs. These high rates suggest that all massive stars (greater than $M_{\text {low }}$ ) give rise to remnants with magnetar-like fields.

This raises these issues: (i) how do all progenitors with $M \geq M_{\text {low }}$ generate $>10^{14} \mathrm{G}$ fields in their compact remnants? and (ii) why is there a dichotomy in magnetic field strength between compact remnants from progenitors with 
mass greater than $M_{\text {low }}$ (i.e., $B \sim 10^{14} \mathrm{G}$ ) and those with mass less than $\sim M_{\text {low }}\left(B \sim 10^{12} \mathrm{G}\right)$ ?

In this study, we introduce the notion of delayed magnetic field amplification as a plausible solution to these issues. We propose that neutron stars from progenitors with mass $M>9 M_{\odot}$ are born with normal $\left(\sim 10^{12} \mathrm{G}\right)$ magnetic fields. A neutron star from a progenitor with an approximate mass in range $M_{\text {low }}<M<60 M_{\odot}$ will experience an explosive transition to a quark star (the QN) in which its magnetic field is amplified to $10^{14}-10^{15} \mathrm{G}$ by color ferromagnetism [32] The transition occurs with a delay of several hundred years [35]. This delayed amplification can in principle alleviate many difficulties in interpreting simultaneously the high birth rate and high magnetic fields of AXPs/SGRs and their link to massive stars. Delayed amplification also might explain the possible discrepancy between spin-down and SNR ages for AXPs/SGRs.

\section{Acknowledgments}

The authors thank the referee (A. D. Kaminker) for helpful suggestions. This research is supported by grants from the Natural Science and Engineering Research Council of Canada (NSERC).

\section{References}

[1] B. M. Gaensler, E. V. Gotthelf, and G. Vasisht, "A New supernova remnant coincident with the slow X-ray pulsar AX J1845-0258," The Astrophysical Journal, vol. 526, p. L37, 1999.

[2] F. P. Gavriil, V. M. Kaspi, and P. M. Woods, "Magnetar-like Xray bursts from an anomalous X-ray pulsar," Nature, vol. 419, no. 6903, pp. 142-144, 2002.

[3] R. Gill and J. Heyl, "The birthrate of magnetars," Monthly Notices of the Royal Astronomical Society, vol. 381, no. 1, pp. 52-58, 2007.

[4] D. Marsden, R. E. Lingenfelter, R. E. Rothschild, and J. C. Higdon, "Nature versus nurture: the origin of soft gammaray repeaters and anomalous X-ray pulsars," The Astrophysical Journal, vol. 550, no. 1, part 1, pp. 397-409, 2001.

[5] B. M. Gaensler, P. O. Slane, E. V. Gotthelf, and G. Vasisht, "Anomalous X-ray pulsars and soft gamma-ray repeaters in supernova remnants," The Astrophysical Journal, vol. 559, no. 2, part 1, pp. 963-972, 2001.

[6] Ü. Ertan, E. Göğüş, and M. A. Alpar, "X-ray and infrared enhancement of anomalous X-ray pulsar IE 2259+586," The Astrophysical Journal, vol. 640, no. 1, pp. 435-440, 2006.

[7] M. A. Alpar, "On young neutron stars as propellers and accretors with conventional magnetic fields," The Astrophysical Journal, vol. 554, no. 2, part 1, pp. 1245-1254, 2001.

[8] P. Chatterjee, L. Hernquist, and R. Narayan, "An accretion model for anomalous X-ray pulsars," The Astrophysical Journal, vol. 534, no. 1, part 1, pp. 373-379, 2000.

[9] R. Ouyed, D. Leahy, and B. Niebergal, "Quark-nova remnants. II. The degenerate torus case with applications to AXPs," Astronomy and Astrophysics, vol. 475, no. 1, pp. 63-70, 2007.

[10] R. Ouyed, D. Leahy, and B. Niebergal, "Quark-nova remnants. I. The leftover debris with applications to SGRs, AXPs, and XDINs," Astronomy and Astrophysics, vol. 473, no. 2, pp. 357368, 2007.
[11] W. H. Press, et al., Numerical Recipes, Cambridge University Press, Chicago, Ill, USA, 1989.

[12] R. N. Manchester, G. B. Hobbs, A. Teoh, and M. Hobbs, "The Australia telescope national facility pulsar catalogue," Astronomical Journal, vol. 129, no. 4, pp. 1993-2006, 2005.

[13] F. Camilo, et al., "The variable radio-to-X-ray spectrum of the magnetar XTE J1810-197,” The Astrophysical Journal, vol. 666, p. L93, 2007.

[14] D. Frail, G. Vasisht, and S. R. Kulkarni, "The changing stucture of the radio nebula around the soft gamma-ray repeater SGR 1806-20," The Astrophysical Journal, vol. 480, p. L29, 1997.

[15] N. M. McClure-Griffiths and B. M. Gaensler, "Constraints on the distance to SGR 1806-20 from H I absorption," The Astrophysical Journal, vol. 630, p. L161, 2005.

[16] G. K. Vasisht, S. R. Kulkarni, D. A. Frail, and J. Greiner, "Supernova remnant candidates for the soft gamma-ray repeater 1900+14," The Astrophysical Journal, vol. 431, p. L35, 1994.

[17] D. R. Lorimer and K. M. Xilouris, "PSR J1907+0918: a young radio pulsar near SGR $1900+14$ and G42.8+0.6," The Astrophysical Journal, vol. 545, no. 1, part 1, pp. 385-389, 2000.

[18] D. A. Smith, H. V. Bradt, and A. M. Levine, "Rossi X-ray timing explorer all-sky monitor localization of SGR 1627-41," The Astrophysical Journal, vol. 519, p. L147, 1999.

[19] F. P. Gavriil and V. M. Kaspi, "Anomalous X-ray rulsar 1E 1048.1-5937: pulsed flux flares and large torque variations," The Astrophysical Journal, vol. 609, p. L67, 2004.

[20] F. Camilo, I. Cognard, S. M. Ransom, et al., "The magnetar XTE J1810-197: variations in torque, radio flux density, and pulse profile morphology," The Astrophysical Journal, vol. 663, no. 1, pp. 497-504, 2007.

[21] R. Kothes, B. Uyaniker, and A. Yar, "The distance to supernova remnant CTB 109 deduced from its environment," The Astrophysical Journal, vol. 576, no. 1, pp. 169-175, 2002.

[22] P. Mészáros, High-Energy Radiation from Magnetized Neutron Stars, University of Chicago Press, Chicago, Ill, USA, 1992.

[23] A. G. Lyne, R. S. Pritchard, F. Graham-Smith, and F. Camilo, "Very low braking index for the Vela pulsar," Nature, vol. 381, no. 6582, pp. 497-498, 1996.

[24] S. Park, D. N. Burrows, G. P. Garmire, J. A. Nousek, J. P. Hughes, and R. M. Williams, "SNR N49 in the LMC: X-ray emission from multi-phase shock and neutron star," Advances in Space Research, vol. 33, no. 4, pp. 409-415, 2004.

[25] N. K. Spyrou and N. Stergioulas, "Spin-down of relativistic stars with phase transitions and PSR J0537-6910," Astronomy and Astrophysics, vol. 395, no. 1, pp. 151-160, 2002.

[26] D. Bhattacharya and V. Soni, "A natural explanation for magnetars," http://arxiv.org/abs/0705.0592.

[27] V. Petit, G. A. Wade, L. Drissen, and T. Montmerle, "Exploring the origin of neutron star magnetic field: magnetic properties of the progenitor OB stars," in Proceedings of the 40 Years of Pulsars-Millisecond Pulsars, Magnetars and More, vol. 983, pp. 399-403, Montreal, Canada, August 2007.

[28] L. Ferrario and D. Wickramasinghe, "Modelling of isolated radio pulsars and magnetars on the fossil field hypothesis," Monthly Notices of the Royal Astronomical Society, vol. 367, no. 3, pp. 1323-1328, 2006.

[29] C. Thompson and R. C. Duncan, "Neutron star dynamos and the origins of pulsar magnetism," The Astrophysical Journal, vol. 408, no. 1, pp. 194-217, 1993.

[30] J. Vink and L. Kuiper, "Supernova remnant energetics and magnetars: no evidence in favour of millisecond protoneutron stars," Monthly Notices of the Royal Astronomical Society, vol. 370, p. L14, 2006. 
[31] A. Heger, S. E. Woosley, and H. C. Spruit, "Presupernova evolution of differentially rotating massive stars including magnetic fields," The Astrophysical Journal, vol. 626, no. 1, pp. 350-363, 2005.

[32] A. Iwazaki, "Color ferromagnetism of quark matter: a possible origin of a strong magnetic field in magnetars," Physical Review D, vol. 72, no. 11, Article ID 114003, 4 pages, 2005.

[33] G. K. Savvidy, "Infrared instability of the vacuum state of gauge theories and asymptotic freedom," Physics Letters B, vol. 71, p. 113, 1977.

[34] A. Iwazaki, O. Morimatsu, T. Nishikawa, and M. Ohtani, "Quantum Hall states of gluons in dense quark matter," Physical Review D, vol. 71, no. 3, Article ID 034014, 12 pages, 2005.

[35] J. E. Staff, R. Ouyed, and P. Jaikumar, "Quark deconfinement in neutron star cores: the effects of spin-down," The Astrophysical Journal, vol. 645, p. L145, 2006.

[36] I. Bombaci, I. Parenti, and I. Vidaña, "Quark deconfinement and implications for the radius and the limiting mass of compact stars," The Astrophysical Journal, vol. 614, no. 1, pp. 314-325, 2004.

[37] R. Ouyed, J. Dey, and M. Dey, "Quark-Nova," Astronomy \& Astrophysics, vol. 390, p. L39, 2002.

[38] P. Keränen and R. Ouyed, "Planets orbiting Quark Nova compact remnants," Astronomy \& Astrophysics, vol. 407, p. L51, 2003.

[39] P. Keränen, R. Ouyed, and P. Jaikumar, "Neutrino emission and mass ejection in quark novae," The Astrophysical Journal, vol. 618, no. 1, pp. 485-492, 2005.

[40] D. Leahy and R. Ouyed, "Supernova SN2006gy as a first ever Quark Nova?" Monthly Notices of the Royal Astronomical Society, vol. 387, no. 3, pp. 1193-1198, 2008.

[41] R. Ouyed, Ø. Elgarøy, H. Dahle, and P. Keränen, "Meissner effect and vortex dynamics in quark stars a model for soft gamma-ray repeaters," Astronomy and Astrophysics, vol. 420, no. 3, pp. 1025-1032, 2004.

[42] R. Ouyed, B. Niebergal, W. Dobler, and D. Leahy, "Threedimensional simulations of the reorganization of a quark star's magnetic field AS induced by the meissner effect," The Astrophysical Journal, vol. 653, no. 1, pp. 558-567, 2006.

[43] B. Niebergal, R. Ouyed, and D. Leahy, "Magnetic field decay and period evolution of anomalous X-ray pulsarsin the context of quark stars," The Astrophysical Journal, vol. 646, p. L17, 2006.

[44] B. Niebergal, R. Ouyed, and D. Leahy, "SGRs and AXPs proposed as ancestors of the magnificent seven," Astronomy \& Astrophysics, vol. 476, p. L5, 2007.

[45] A. Dar and A. De Rújula, "SGRs and AXPs - magnetars or young quark stars?" in Results and Perspectives in Particle Physics, M. Greco, Ed., vol. 17, p. 13, 2000.

[46] R. Ouyed, D. Leahy, B. Niebergal, and Y. Yue, "Quark-nova remnants. III. Application to RRATs," Monthly Notices of the Royal Astronomical Society, vol. 396, no. 2, pp. 1058-1065, 2009. 

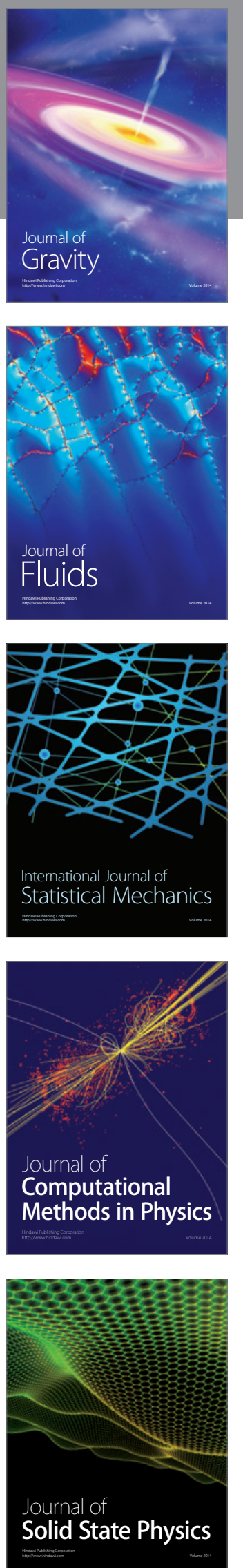

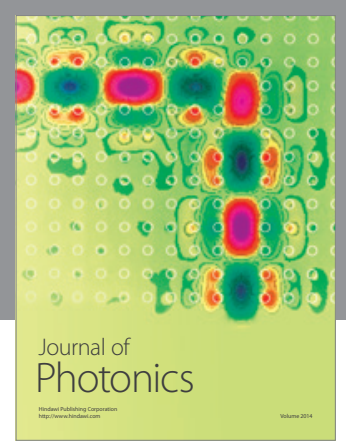

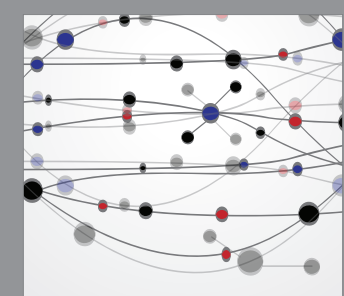

The Scientific World Journal
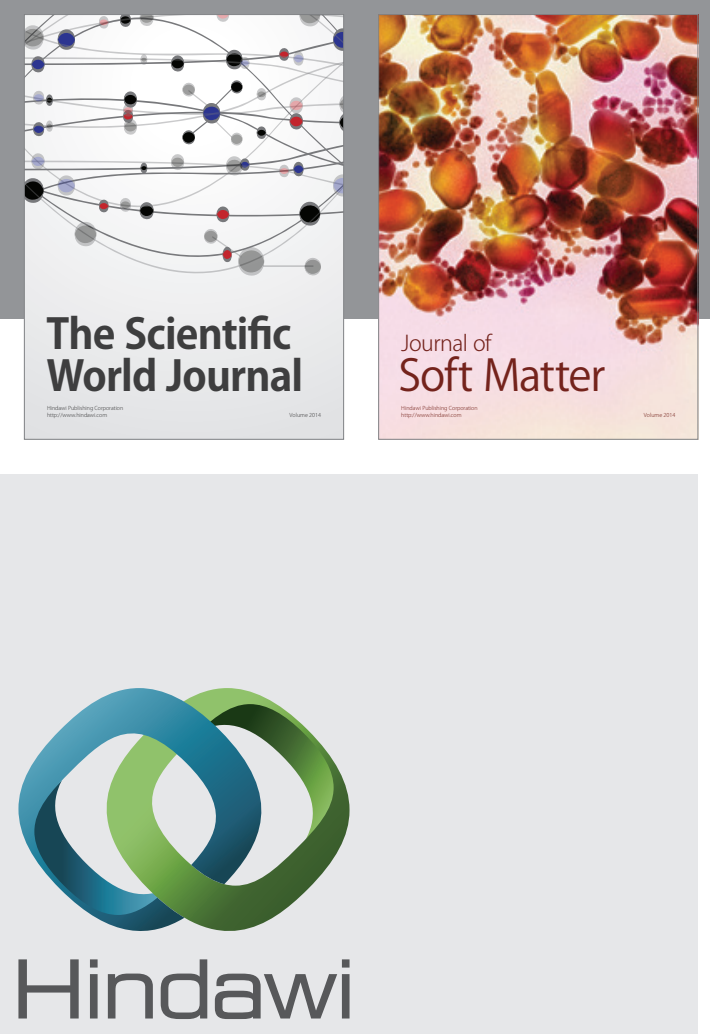

Submit your manuscripts at

http://www.hindawi.com
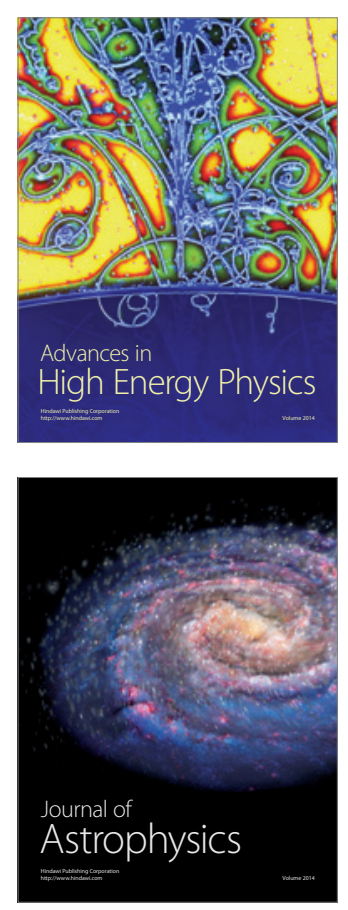
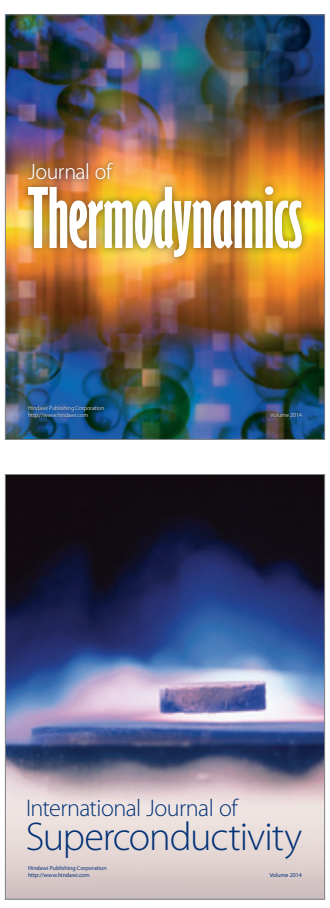
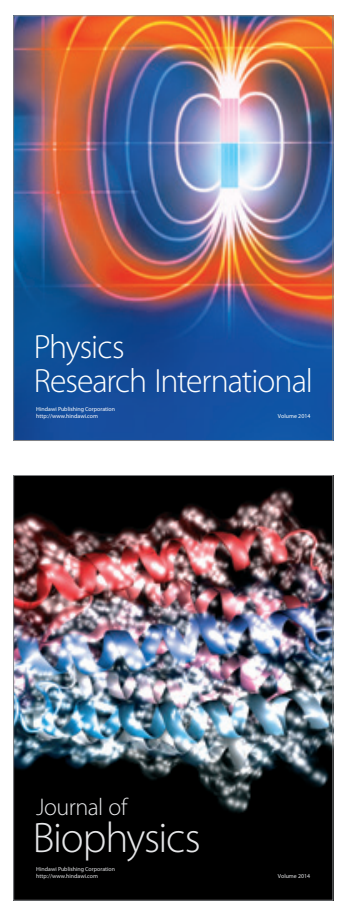
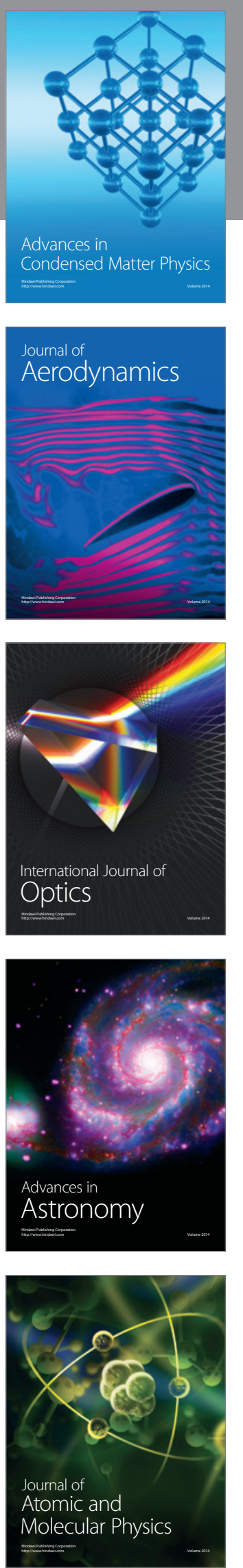